\title{
Olfactory Reference Syndrome: A Case Report and Screening Tool
}

Yelena Chernyak, $1 \square$

Email ychernya@iupui.edu

Kristine M. Chapleau, 1

Shariff F. Tanious, 1

Natalie C. Dattilo, 2

David R. Diaz, 1

Sarah A. Landsberger, 1 
1 Department of Psychiatry, Indiana University School of Medicine, 355 W. 16th St., Suite 2800, Indianapolis, IN, 46202 USA

2 Department of Psychiatry, Brigham and Women's

Hospital, Boston, MA, USA

\section{Abstract}

Olfactory reference syndrome (ORS) is a lesser known disorder that is related to obsessive-compulsive disorder. ORS is the obsessional and inaccurate belief that one is emitting a foul odor leading to embarrassment or concern about offending others, excessive hygiene behaviors, and social avoidance that significantly interferes with daily functioning. Although ORS is rare, it is challenging to diagnose. ORS-sufferers first seek treatment from non- psychiatric providers (e.g., dermatologists, dentists.) to alleviate the perceived odor, which frequently leads to misdiagnosis and unnecessary treatments. Additionally, because ORSsufferers can have limited insight and ideas of reference, they can be misdiagnosed as having a psychotic or delusional disorder. We present a case report of a 42-year-old woman with ORS, and how the correct diagnosis of ORS provided with psychiatric treatment led to significant improvement in her daily functioning. We provide a literature review on the disorder as well as a short screener to assess ORS.

AQ1

AQ2

\section{Keywords}

Olfactory reference syndrome

Psychiatric disorders

Ethics

Anxiety disorders

Delusional disorders

Obsessive-compulsive disorder

Jikoshu-kyofu

Taijin-kyofu 


\section{Introduction}

A lesser known obsessive-compulsive-related disorder is olfactory reference syndrome (ORS). A person with ORS is preoccupied with the belief that he or she emits an offensive body odor, even though no such odor is detected by others (Phillips \& Menard, 2011). This preoccupation causes clinically significant distress or impairment in daily functioning; specifically large amounts of time spent engaging in repetitive and excessive behaviors to camouflage the perceived odor, and avoidance of social situations that are feared to be embarrassing (Feusner, Phillips, \& Stein, 2010). ORS and its symptoms are frequently debilitating to the patient. Some have considered this syndrome a variant of social anxiety disorder due to the focus on social evaluation (Lochner \& Stein, 2003). ORS had been previously categorized as a delusional disorder in the Diagnostic and Statistical Manual of Mental Disorders, Fourth Edition, Text Revision (DSMIV-TR; APA, 2000). It has since been re-categorized as a syndrome analogous with jikoshu-kyofu, another specified obsessive-compulsive and related disorder in DSM, Fifth Edition (DSM-5; APA, 2013).

Most ORS-sufferers first seek treatment from dermatologists, dentists, proctologists, and otolaryngologists to alleviate the perceived odor (Greenberg, Shaw, Reuman, Schwartz, \& Wilhelm, 2016; Lochner \& Stein, 2003; Phillips \& Menard, 2011). Just as ORS-sufferers are dissatisfied with their own efforts to mask the perceived odor, standard medical interventions for the patient's perceived symptoms will also produce little to no relief (Teraishi et al., 2012). ORS-sufferers are at risk for receiving (and overusing) unnecessary medications and prescriptionlevel products, injections, and surgical interventions (Greenberg, et al., 2016; Phillips \& Menard, 2011). Continued failed non- psychiatric attempts to correct the perceived odor can lead to feelings of hopelessness and suicidal ideation (Phillips \& Menard, 2011). Therefore, although ORS is rare (Kasahara \& Kenji, 1971; Marks \& Mishan, 1988), early identification is important (Teraishi et al., 2012).

\section{Case Study}

A 42-year-old African American woman was referred to an outpatient psychiatric clinic with depressed mood and periodic suicidal ideation. The patient had been on work disability for several years due to depressive symptoms, was never 
married, had no children, and had little social contact. She reported being harassed by others, which led to her increased social isolation, being homebound for several days at a time, and eventual abandonment of educational and career goals. When the patient did leave her home, she reported that others were visibly offended by her foul body odor, which she described as having a "musty" smell and at times smelling like "boiled meat" or stool, emanating from the axillary, genital, or chest areas. Examples of such harassment included a store patron who stood next to her and "sniffed" to identify the source of the foul odor; a woman on a bus who was sitting nearby covered her nose and mouth; a co-worker left a sponge on the patient's chair signifying to patient that she should "clean herself up," and overheard remarks from passers-by ("She smells."). She described these incidents as profoundly embarrassing and anxiety-provoking, causing her to exit the situation at first opportunity. She attempted to camouflage the perceived odor by engaging in numerous repetitive behaviors: she showered twice a day, tucked tissue under her clothing to absorb sweat and moisture, applied antiperspirant approximately 40 times per underarm, laundered clothing after one wearing, and inserted scented desiccate packets in her shoes during wear. Onset of symptoms was 11 years old. As a child, patient reported that she attended a predominantly White school and was severely bullied for having epileptic seizures (which she no longer had nor was taking medication to control) and body odor.

Before seeking psychiatric care, she continuously sought dermatological treatment for hyperhidrosis and bromhidrosis. Patient did meet diagnostic criteria for hyperhidrosis: focal, visible sweating with impaired daily activities, at least $1 \times /$ week, onset younger than 25 years, and she had a family history of hyperhidrosis (but not bromhidrosis), and cessation of focal sweating during sleep. Prescription-strength antiperspirants, curettage of the sympathetic system, and Drionic units were tried; however, none provided satisfactory relief and in fact, post-curettage the patient reported that her sweating was worse. She also reported having dry skin which was exacerbated by frequent and harsh cleaning.

The patient periodically sought mental health care but was repeatedly misdiagnosed with schizoaffective disorder or schizotypal personality disorder, resulting in her disengagement from care. Upon the present evaluation, psychotic disorders were ruled out because there was no evidence of positive or negative symptoms. Rather, she presented as interpersonally engaging, well-groomed and appropriately dressed, with good eye contact and normal range of affect that was 
congruent with emotion. Olfactory reference syndrome (with poor insight) was diagnosed and patient was provided psychoeducation on the disorder, emphasizing that ORS is related to obsessive-compulsive disorder. Although the patient was still convinced that the odor existed, the correct diagnosis provided a great deal of relief for her and increased her engagement in treatment and subsequently increased her family support and involvement.

Psychotherapy, medication management, and a neurology consult were recommended for the treatment of this patient. Patient was assessed by a neurologist and there was no indication of current seizure activity or other neurological contributor to her symptoms. She was prescribed fluoxetine, titrated up to $60 \mathrm{mg}$, and provided with medication education. Psychotherapy utilized behavioral exposure-based techniques which increased the patient's exposure to social risk and avoided situations without compulsive hygiene behaviors; social factors such as the patient's experience of racism as it related to her symptoms were also discussed. Two years after receiving the diagnosis of ORS, the patient still struggled with ideas of reference, but her functioning was markedly improved. With treatment, she was able to complete her education, had returned to work, and reestablished a supportive relationship with family members.

\section{Discussion}

\section{Etiology, Prevalence, and Clinical Features}

Although the causes of ORS are unknown, ORS is likely due to a combination of neurobiological, genetic, behavioral, cognitive, and environmental factors (Begum \& McKenna, 2011). As with OCD, the neurobiological mechanism is likely a circuit in the brain that relays information from the orbitofrontal cortex to the striatum and thalamus. When this circuit is active, one's thoughts and urges become a focus of attention which compels behaviors to neutralize the unwelcome thought or urge. Specific to ORS, negative experiences related to smell, such as a classmate teasing the individual about body odor as illustrated in the case study, may trigger the development of ORS in predisposed individuals.

Estimates of ORS range from 0.5 to $2.1 \%$ (Kasahara \& Kenji, 1971; Marks \& Mishan, 1988). It is more prevalent in men although greater symptom severity and worse insight has been reported in women (Bizamcer, Dubin, \& Hayburn, 2008; Greenberg, et al., 2016). Age of onset is 16-26 years old which worsens 
over time in $>50 \%$ of cases (Greenberg et al., 2016).

In the largest study of people with ORS $(N=253)$, Greenberg et al. (2016) found that the most common symptom was the preoccupation of bad smells emanating from two or more body areas (e.g., armpits, feet, breasts). Smells could be somatic (i.e., sweat, stool) or non-somatic (e.g., garbage, ammonia, metal, or stool from an unlikely source), and preoccupation with non-somatic smells is associated with neurological conditions such as migraine headaches and seizure disorders. Almost all performed compulsive behaviors 3-8 h per day to mask or correct the perceived odor, most commonly excessive showering and deodorant use. Additionally, the majority had olfactory hallucinations (59\%) as well as ideas of reference $(64 \%)$ in which the individual misinterprets others' innocuous gestures (e.g., sniffing, throat clearing, opening a window, laughing, as the case illustrates) as confirmation that his or her body odor is detectable and offensive, evoking intense embarrassment, and shame. Almost all avoided social situations and were housebound for 1.5-7.5 consecutive days each month, which led to high absenteeism at work or school.

\section{Differential Diagnosis of ORS and Other Medical Conditions}

ORS patients most often complain of axillary, plantar, and/or genital bromhidrosis and hyperhidrosis. Upon physical examination, body odor may be absent or far less noticeable than the patient's report, and the patient may dismiss such feedback, as with our case study. ORS should be considered when the patient complains that, despite excessive hygiene practices, malodor is unremitting or returns suddenly at variable times as opposed to malodor reliably emerging over time as hygiene products wear off. Still, hyperhidrosis can cooccur with ORS and should be treated if present. Skin conditions, such as dermatitis, may also be present due to irritation from excessive and improper use of hygiene products.

\section{Differential Diagnosis of ORS and Other Psychiatric Conditions}

ORS symptoms overlap with several, more well-known disorders: obsessivecompulsive disorder, social anxiety disorder and, when insight is absent, delusional disorder-somatic type (Feusner et al., 2010). ORS was once thought to be a culture-bound disorder limited to Japan and Korea (taijin-kyofushu or 
jikoshu-kyofu), but has been found across cultures (Feusner et al., 2010; Stein et al., 1998). The perceived lack of control over body odor and social interactions contribute to lower mood. Almost all ORS patients had comorbid depression $(97 \%)$, as well as suicidal ideation (68\%) and attempted suicide (32\%; Suzuki et al., 2004).

The ICD-11 Working Group on Classification for Obsessive Compulsive Related Disorders reclassified ORS as an obsessive-compulsive-related disorder rather than a delusional disorder for two reasons. Some ORS patients can have good-tofair insight, unlike the case presented above, and ORS has common clinical features with obsessive compulsive disorder (OCD) and obsessive compulsiverelated disorders ().

There are many parallels between the symptoms of ORS and OCD. Specifically, the intrusive and troubling thoughts and the repetitive and compulsive behaviors map on to the symptomatology of OCD. However, unlike ORS, OCD beliefs are delusional in less than $5 \%$ of cases and ideas of reference are less common (Kozak \& Foa, 1994).

ORS is also similar to body dysmorphic disorder (BDD) and body-focused repetitive disorders (i.e., trichotillomania and skin excoriation). All disorders involve preoccupation with a perceived bodily defect, although ORS differs in that the preoccupation is with odor rather than with physical appearance and/or tactile sensations. Preoccupation leads to anxiety, avoidance of social situations, and compulsive behaviors such as checking and time spent attempting to mask or correct the perceived defect. Patients with these disorders seek medical interventions such as surgical, dermatological, and dental treatment for the perceived defect and are dissatisfied with the results (Lochner \& Stein, 2003). Indeed, most (85\%) ORS patients sought non-psychiatric treatment to fix the perceived malodor (see also Begum \& McKenna, 2011; Greenberg et al., 2016; Phillips \& Menard, 2011; Pryse-Phillips, 1971; Teraishi et al., 2013; Stein, Le Roux, Bouwer, \& Van Heerdeen, 1998).

\section{AQ3}

ORS symptoms overlap with delusional disorders of the somatic type if insight is absent. Insight can range from good (lingering doubts), to fair (unrealistic fears), to poor (overvalued ideas), to absent (delusional; see Phillips \& Menard, 2011). 
Only the minority of ORS patients (18\%) have poor or absent insight (Greenberg et al., 2016). Still, worse insight is associated with longer symptom duration, more treatment resistance, more physician consultations, and greater loss of sociooccupational functioning (Lim \& Wan, 2008).

\section{Assessment and Treatment Considerations}

Currently, there are two clinician-rated scales used to assess ORS: The Social Anxiety/Taijin-Kyofu Scale (SATS; Asakura et al., 2012) and The Yale-Brown Obsessive Compulsive Scale Modified for ORS (ORS-YBOCS) adapted from the YBOCS for Body Dysmorphic Disorder (Phillips, Hollander, Rasmussen, Aronowitz, DeCaria, \& Goodman, 1997; Phillips \& Menard, 2011). Both the SATS and the ORS-YBOCS contain 12 items. Given the length and accessibility of these scales, providers may wish to use the 3 -item ORS screening questions provided in Fig. 1.

\section{Fig. 1}

Olfactory reference syndrome screening questions

\section{Brief Screening Test for Olfactory Reference Syndrome}

Offending others: Do you fear that your body odor offends others?

Reference: Can you tell by others' gestures or behaviors that they are offended by your body odor?

Social avoidance: Because of your body odor do you avoid social situations to the point that it interferes with your life or is disabling?

Adapted from the Social Anxiety/Taijin-Kyofu Scale (SATS) Supplement. (Available at: http://www.karger.com/ProdukteDB/miscArchiv/000/329/741/000329741_sm/html)

With respect to psychiatric treatment for ORS, the literature consists only of case reports and small case series; there are no randomized controlled trials of treatment options. However, as with other obsessive-compulsive-related disorders, ORS appears to respond to SSRIs alone (Begum \& McKenna, 2011; 
Bizamcer et al., 2008; Stein et al., 1998; Teraishi et al., 2012). SSRIs plus an antipsychotic also showed efficacy in some cases (Kozak \& Foa, 1994). There is less evidence that antipsychotic monotherapy helps unless there is clear evidence of a co-occurring psychotic disorder (Prazeres et al., 2010). Case studies have reported improvement with psychotherapy alone (Allen-Crooks \& Challacombe, 2017), with the greatest improvement with combined SSRIs and psychotherapy (Begum \& McKenna, 2011).

\section{Conclusion}

ORS is likely under-identified in patients presenting for medical care. When the presence of ORS is suspected, physicians should screen the patient for symptoms of ORS, educate the patient about the suspected diagnosis and make an appropriate referral to a mental health provider for additional assessment and treatment. Although evidence is limited, a combination of SSRI treatment and cognitive-behavioral psychotherapy is recommended. Education and concern provided by physicians may help patients increase insight or willingness to seek out psychiatric care. At minimum, identification of ORS by physicians and appropriate psychiatric referral would prevent unnecessary, costly, and possibly risky medical treatment, as well as reinforcement of the patient's psychiatric symptoms. More research is needed on the course and effective treatment of ORS. Specifically, large randomized controlled trials are needed, but given the rarity of ORS this presents a formidable challenge for researchers.

\section{AQ4}

\section{Publisher's Note}

Springer Nature remains neutral with regard to jurisdictional claims in published maps and institutional affiliations.

\section{Funding}

This article has no funding source.

\section{Compliance with Ethical Standards}

Conflict of Interest Yelena Chernyak, Kristine M. Chapleau, Shariff F. Tanious, Natalie C. Dattilo, David R. Diaz, and Sarah A. Landsberger declare that they have no conflict of interest. 
Informed Consent Informed consent was obtained from all individual participants included in this case report.

\section{References}

Allen-Crooks, R., \& Challacombe, F. (2017). Cognitive behavior therapy for olfactory reference disorder (ORD): A case study. Journal of ObsessiveCompulsive and Related Disorders, 13, 7-13.

American Psychiatric Association. (2000). Diagnostic and Statistical Manual of Mental Disorders: DSM-IV-TR. Washington, DC: APA.

American Psychiatric Association. (2013). Diagnostic and Statistical Manual of Mental Disorders, DSM-5. Washington, DC: APA.

Asakura, S., Inoue, T., Kitagawa, N., Nasegama, M., Fujii, Y., Kako, Y., ... Nakagawa, S. (2012). Social Anxiety/Taijin-Kyofu Scale (SATS): Development and psychometric evaluation of a new instrument.

Psychopathology, 45, 96-101.

Atmaca, M., Korkmaz, S., Namli, M. N., Kormaz, H., \& Kuloglu, M. (2011). Olfactory reference syndrome treated with quetiapine: A case. Bull of Clinical Psychopharmacology, 21, 246-248.

Begum, M., \& McKenna, P. J. (2011). Olfactory reference syndrome: A systematic review of the world literature. Psychological Medicine, 41, 453461.

Bizamcer, A. N., Dubin, W. R., \& Hayburn, B. (2008). Olfactory reference syndrome. Psychosomatics, 49, 77-81.

Feusner, J. D., Phillips, K. A., \& Stein, D. J. (2010). Olfactory reference syndrome: Issues for DSM-V. Depression \& Anxiety, 27, 592-599.

Greenberg, J. L., Shaw, A. M., Reuman, L., Schwarts, R., \& Wilhelm, S. (2016). Clinical features of olfactory reference syndrome: An internet-based study. Journal of Psychosomatic Research, 80, 11-16. 
Kasahara, Y., \& Kenji, S. (1971). Ereuthophobia and allied conditions: A contribution toward the psychopathological and cross cultural study of a borderline state. In S. Arieti (Ed.), The world biennial of psychiatry in psychotherapy. New York: Basic Books.

Kozak, M. J., \& Foa, E. B. (1994). Obsessions, overvalued ideas, and delusions in obsessive-compulsive disorder. Behavior Research and Therapy, $32,343-353$.

Lim, L., \& Wan, Y. M. (2015). Jikoshu-kyofu in Singapore. Australasian Psychiatry, 23, 300-302.

Lochner, C., \& Stein, D. (2003). Olfactory reference syndrome: Diagnostic criteria and differential diagnosis. Journal of Postgraduate Medicine, 49, 328331.

Marks, I., \& Mishan, J. (1988). Dysmorphophobic avoidance with disturbed bodily perception: A pilot study of exposure therapy. British Journal of Psychiatry, 152, 674-678.

Muffatti, R., Scarone, S., \& Gambini, O. (2008). An olfactory reference syndrome successfully treated by aripiprazole augmentation to antidepressant therapy. Cognitive and Behavioral Neurology, 21, 258-260.

Phillips, K. A., Hollander, E., Rasmussen, S. A., Aronowitz, B. R., DeCaria, C., \& Goodman, W. K. (1997). A severity rating scale or body dysmorphic disorder: Development, reliability, and validity of a modified version of the Yale-Brown Obsessive Compulsive Scale. Psychopharmacology Bulletin, 33, $17-22$.

Phillips, K. A., \& Menard, W. (2011). Olfactory reference syndrome: Demographic and clinical features of imagined body odor. General Hospital Psychiatry, 33, 398-406.

Prazeres, A. M., Fontenelle, L. F., Medlowicz, M. V., De Mathis, M. A., Ferraro, Y. A., de Brito, N. F., ... Miguel, E. C. (2010). Olfactory reference syndrome as a subtype of body dysmorphic disorder. Journal of Clinical 
Psychiatry, 71, 87-89.

Pryse-Phillips, W. (1971). An olfactory reference syndrome. Acta Psychiatrica Scandinavica, 47, 484-509.

Stein, D. J., Le Roux, L., Bouwer, C., \& Van Heerdeen, B. (1998). Is olfactory reference syndrome an obsessive-compulsive spectrum disorder?: Two cases and a discussion. Journal of Neuropsychiatry and Clinical Neuroscience, 10, 96-99.

Stein, D. J., Kogan, C. S., Atmaca, M., Fineberg, N. A., Fontenelle, L. F., Grant, J. E., ... Van Den Heuvel, O. A. (2016). The classification of obsessive-compulsive and related disorders in the ICD-11. Journal of Affective Disorders, 190, 663-674.

Suzuki, K., Takei, N., Iwata, Y., Sekine, Y., Toyoda, T., Nakamura, K., ... Mori, N. (2004). Do olfactory reference syndrome and jiko-shu-kyofu (a subtype of taijin-kyofu) share a common entity? Acta Psychiatrica Scandinavica, 109, 150-155.

Teraishi, T., Takahashi, T., Suda, T., Hirano, J., Ogawa, T., Kuwahara, T., ... Nomura, S. (2012). Successful treatment of olfactory reference syndrome with paroxetine. Journal of Neuropsychiatry and Clinical Neuroscience, 24, E24. 